\section{Chronischer Schmerz nach Fraktur}

Ein Knochenbruch prädisponiert offenbar dazu, ausgedehnte chronische Schmerzen zu entwickeln, wie eine englische Studie zeigt. Gebrochene Knochen erhöhten das Risiko für CWP (Chronic Widespread Pain) signifikant. Stark ausgeprägt war die Steigerung bei Wirbelbrüchen mit einem höheren Risiko von 2,7 bei Männern und 2,1 bei Frauen. Hüftfrakturen waren bei Frauen ebenfalls Gefährdungsfaktor (2,2). Der kausale Zusammenhang müsse aber noch geklärt werden, so die Autoren.

Walker-Bone Ket al. Arch Osteoporos 2016;11:1

\section{Kartoffeln - ein Risiko für Schwangere?}

Glaubt man US-Epidemiologen, sollten Schwangere Kartoffeln meiden. Denn der Konsum der Stärkefrucht steigere das Risiko für einen Gestationsdiabetes. Die Rate war bei einer Portion pro Woche um 20\% erhöht, bei zwei bis vier um $27 \%$ und bei mehr als fünf Portionen um $50 \%$, verglichen mit unter einer Portion. Die Daten entstammen der Nurses' Health Study.

Bao Wet al. BMJ 2016; 352:h6898

\section{Macht Fernsehen dumm?}

Es scheint etwas an dem Klischee dran zu sein: Wer als junger Mensch viel vor der Glotze sitzt (>3 h/Tag), hat später Defizite in der Verarbeitungsgeschwindigkeit und Exekutivfunktion, so das Ergebnis einer USLangzeitstudie. 3250 junge Erwachsene wurden 25 Jahre lang nach TV-Gewohnheiten und Freizeitaktivitäten befragt und unterzogen sich ausführlichen Kognitionstests (DSST, Stroop-Test, RALVT). Wenig Sport war ebenfalls schlecht für die Hirnleistung - wenn auch nicht so dramatisch.

Hoang TD et al. JAMA Psychiatry 2016; 73(1):73-79

Primäre Hyperhidrose

\title{
Oxybutynin stoppt übermäßiges Schwitzen
}

Für Menschen, die übermäßig schwitzen, scheint laut einer aktuellen Studie niedrigdosiertes Oxybutynin eine effektive Therapieoption zu sein.

Dass das Anticholinergikum Oxybutynin auch an den Schweißdrüsen wirkt, ist seit Längerem bekannt. Studien dazu gab es bislang nur wenige. Nun reichen Dermatologen eine zwar kleine, aber randomisierte placebokontrollierte Studie nach: 62 Patienten mit primärer Hyperhidrose wurden in Verum- und Placebogruppe eingeteilt. Die Oxybutyningruppe startete mit einer Tagesdosis von 2,5 mg, die schrittweise auf 7,5 mg pro Tag gesteigert wurde. Die Dosis lag damit unter der Dosierung, die bei der Behandlung der überaktiven Blase üblich ist (10-15 mg).
Nach sechs Wochen zeigte sich Oxybutynin dem Placebo überlegen: $67 \%$ aus der Wirkstoffgruppe, aber nur $27 \%$ unter Placebo gaben an, weniger zu schwitzen. Von den 18 Verumpatienten, die eine Wirkung bestätigten, reduzierte sich der Ausgangswert bei fünf Probanden um einen Punkt auf der Schweregrad-Skala, bei elf um zwei und bei zwei um drei Punkte. Das Placebo besserte den Wert bei zwei Patienten um zwei Punkte und bei sechs um einen Punkt.

Schwerwiegende Nebenwirkungen traten während der sechswöchigen Behandlung nicht auf. Fast die Hälfte der Patienten klagte aber über einen trockenen Mund, in der Placebogruppe drei.

Schollhammer Met al. Oxybutynin as a treatment for generalized hyperhidrosis. BJD 2015; 14. Okt. 2015

\section{Blut raus, Druck runter}

\section{Hilft Aderlass bei arterieller Hypertonie?}

Zwei Fliegen mit einer Klappe: In einer neuen Beobachtungsstudie korrelieren regelmäßige Blutspenden bei hypertensiven Spendern mit einem deutlichen Abfall des Blutdrucks.

Mit „Aderlässen“ werden heute nicht mehr die Spender, sondern die Empfänger behandelt. Könnte sich das wieder ändern? Eine neue Beobachtungsstudie von Transfusions- und Komplementärmedizinern der Charité und des Immanuel-Krankenhauses Berlin sowie der Universität WittenHerdecke lässt aufhorchen. 292 Blutspender, davon 146 mit einem Blutdruck von $>$ 140/90 mmHg, wurden ein Jahr lang hinsichtlich ihres Blutdruckverhaltens beobachtet. Gemessen wurde mehrfach im Sitzen vor und nach der Blutspende. Der Wert wurde gemittelt. Blut gespendet wurde mit unterschiedlicher Frequenz. 114 der hypertensiven Probanden spendeten zweimal, 39 spendeten viermal, also alle drei Monate.

\section{Dosis-Wirkungs-Beziehung}

Nach einem Jahr zeigte sich bei den Spendern mit Hypertonie ein deutlicher Abfall des Blutdrucks, der in der Gruppe der normotensiven Blutspender nicht beobachtet wurde. Am deutlichsten war der Effekt bei jenen Patienten, die alle drei Monate Blut spendeten. Der systolische Blutdruck sank in dieser Gruppe von 156 auf $144 \mathrm{mmHg}$, der diastolische von 91 auf $85 \mathrm{mmHg}$. Änderungen in der Medikation als Begründung wurden ausgeschlossen. Noch sind die Mechanismen dahinter unklar.

(gvg)

Kamhieh-Milz S et al. Regular blood donation may help in the management of hypertension. Transfusion. 2015 Dec 8. 